Provided for non-commercial research and education use. Not for reproduction, distribution or commercial use.

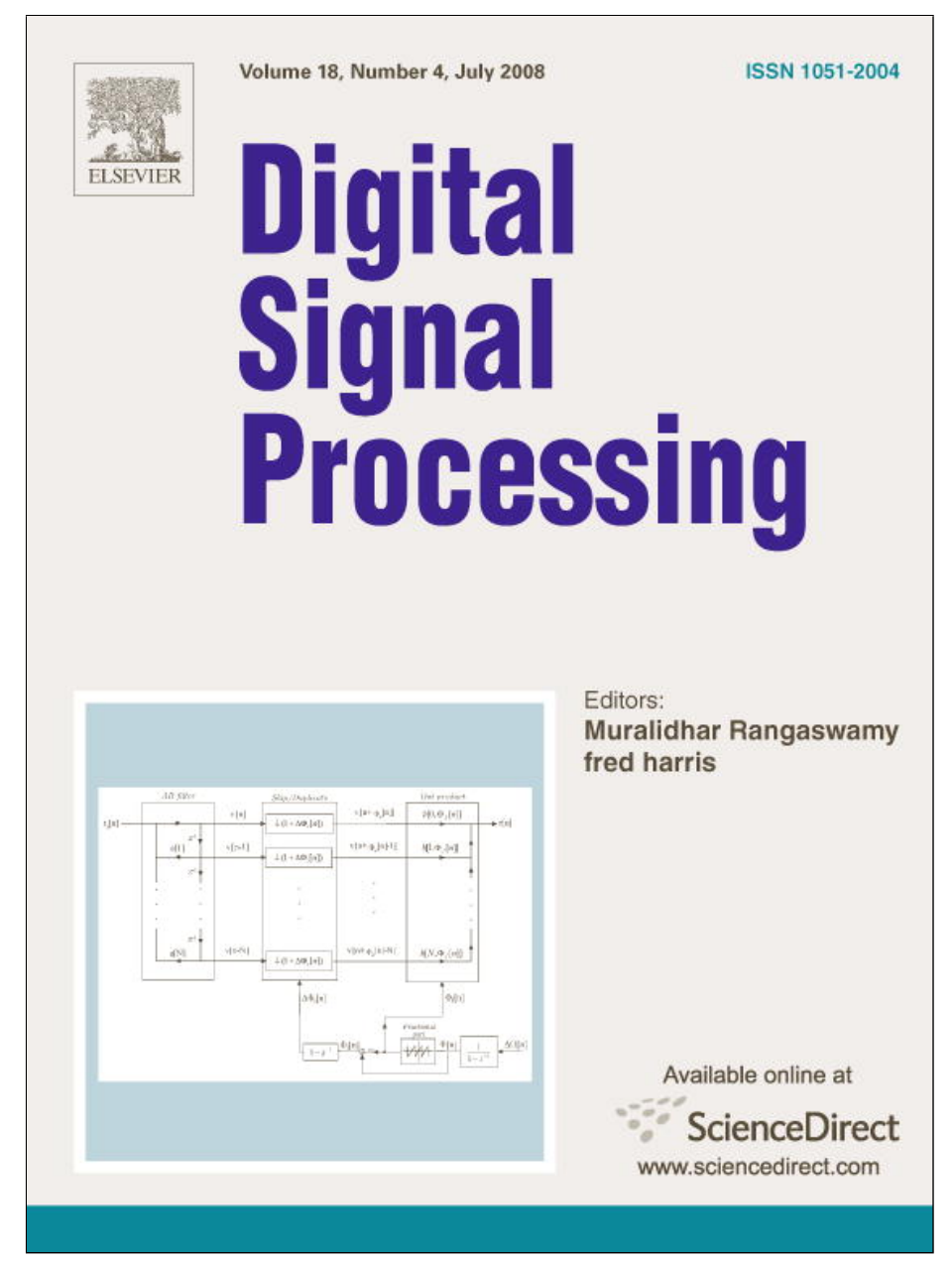

This article appeared in a journal published by Elsevier. The attached copy is furnished to the author for internal non-commercial research and education use, including for instruction at the authors institution and sharing with colleagues.

Other uses, including reproduction and distribution, or selling or licensing copies, or posting to personal, institutional or third party websites are prohibited.

In most cases authors are permitted to post their version of the article (e.g. in Word or Tex form) to their personal website or institutional repository. Authors requiring further information regarding Elsevier's archiving and manuscript policies are encouraged to visit:

http://www.elsevier.com/copyright 


\title{
Adaptive Bayesian space-time equalisation for multiple receive-antenna assisted single-input multiple-output systems
}

\author{
S. Chen*, S. Liu, L. Hanzo \\ School of Electronics and Computer Science, University of Southampton, Southampton SO17 1BJ, UK
}

Available online 3 October 2007

\begin{abstract}
This contribution considers nonlinear space-time equalisation (STE) for multiple receive-antenna induced single-input multipleoutput (SIMO) systems. By exploiting the inherent symmetry of the underlying optimal Bayesian STE solution, a novel symmetric radial basis function (RBF) based STE scheme is proposed, which is capable of approaching the optimal Bayesian equalisation performance. Adaptive implementation of this symmetric RBF (SRBF) based STE can be achieved conveniently by estimating the SIMO channels using the least mean square channel estimator and computing the optimal RBF centres from the resulting SIMO channel matrix estimate. Simulation results also demonstrate that the performance of this SRBF based STE is robust with respect to the choice of the RBF variance value. The proposed adaptive solution is then extended to the space-time decision feedback equalisation (ST-DFE) structure.
\end{abstract}

(c) 2007 Elsevier Inc. All rights reserved.

Keywords: Multiple antennas; Single-input multiple-output; Space-time equaliser; Space-time decision feedback equaliser; Bayesian equaliser; Symmetric radial basis function network

\section{Introduction}

Space-time processing techniques plays an increasingly important role in wireless communications [1-7]. With the aid of smart antenna arrays and by exploiting both the space and time dimensions, space-time processing is capable of effectively improving the achievable system capacity, coverage and quality of service by suppressing both intersymbol interference and co-channel interference. The family of single-input multiple-output (SIMO) systems has enjoyed popularity owing to its simplicity. A space-time equaliser (STE) [8-14] based on the SIMO structure of a single-antenna transmitter and a multiple-antenna receiver is capable of mitigating the channel impairments arising from hostile multipath propagation. The most commonly used STE structure is linear and classically the design of such a linear STE is based on the minimum mean square error criterion. A recent study has shown that the optimal linear STE design is the one that adopts the minimum bit error rate (MBER) criterion [12]. This linear MBER design has been extended to the linear space-time decision feedback equalisation (ST-DFE) structure [13,14].

In this contribution, we show that the true optimal STE solution for the SIMO system is nonlinear, just as in the case of single-input single-output (SISO) systems [15-20]. We further highlight the inherent symmetry of the

\footnotetext{
* Corresponding author. Fax: +44 (0)23 80594508 .

E-mail address: sqc@ecs.soton.ac.uk (S.Chen).
} 
optimal Bayesian STE solution, and this enables us to propose an adaptive nonlinear STE based on a novel symmetric radial basis function (RBF) network. Such a symmetric RBF (SRBF) network was proposed recently for realising the optimal Bayesian equaliser in the SISO case [21]. A convenient way of adaptive implementation of this SRBF STE is to estimate the SIMO channels using the least mean square (LMS) channel estimator and then to compute the optimal RBF centre vectors based on the resulting SIMO channel matrix estimate. This adaptive SRBF STE is capable of realising the optimal Bayesian STE and its performance is robust with respect to the choice of the RBF variance value, as will be demonstrated in a simulation investigation. We then investigate the optimal nonlinear STDFE solution for the SIMO system. By adopting the space-translation interpretation of decision feedback [22], the ST-DFE structure is transformed into a STE structure in the translated observation space, and this enables us to apply the adaptive solution for the STE to the ST-DFE.

The paper is organised as follows. Section 2 briefly presents the SIMO system model as well as the generic STE and ST-DFE structures. The optimal Bayesian STE solution and its adaptive realisation by the SRBF network are derived in Section 3, while Section 4 investigates the optimal Bayesian ST-DFE solution and its adaptive implementation. Simulation results are presented in Section 5, and the paper concludes at Section 6.

\section{SIMO system model and equalisation structure}

A SIMO system employs a single transmit antenna and $L(>1)$ receive antennas, as depicted in Fig. 1, where $s(t)$ is the transmitted signal, $x_{l}(t)$ denotes the $l$ th receiver antenna's output signal, and $n_{l}(t)$ the $l$ th channel's noise. The received signals are sampled at the symbol rate to obtain the $L$ antennas' output samples $x_{l}(k), 1 \leqslant l \leqslant L$, which can be expressed as

$$
x_{l}(k)=\sum_{i=0}^{n_{c}-1} c_{i, l} s(k-i)+n_{l}(k)=\bar{x}_{l}(k)+n_{l}(k),
$$

where $n_{l}(k)$ is a complex-valued Gaussian white noise process with $E\left[\left|n_{l}(k)\right|^{2}\right]=2 \sigma_{n}^{2}, \bar{x}_{l}(k)$ denotes the noise-free part of the $l$ th channel's complex-valued output, the modulation scheme is assumed to be binary phase shift keying (BPSK) and therefore the transmitted symbol sequence $s(k)$ is real-valued and takes values from the BPSK symbol set $\{ \pm 1\}$, and $c_{i, l}$ are the complex-valued taps of the $l$ th channel impulse response (CIR) having a length of $n_{c}$. For notational simplicity, we have assumed that each of the $L$ channels has the same length of $n_{c}$. The proposed nonlinear equalisation structures can be extended to complex-valued modulation schemes, such as quadrature phase shift keying, at the cost of an increase in computational complexity [20,23].

The multiple-channel output samples $x_{l}(k), 1 \leqslant l \leqslant L$, are passed to a generic STE or a generic ST-DFE, as shown in Figs. 2 and 3, respectively. In the generic STE structure of Fig. 2, $M$ is the feedforward temporal filter's order, $d$ denotes the equaliser's decision delay, and $\hat{s}(k-d)$ is the decision for the transmitted symbol $s(k-d)$. For the

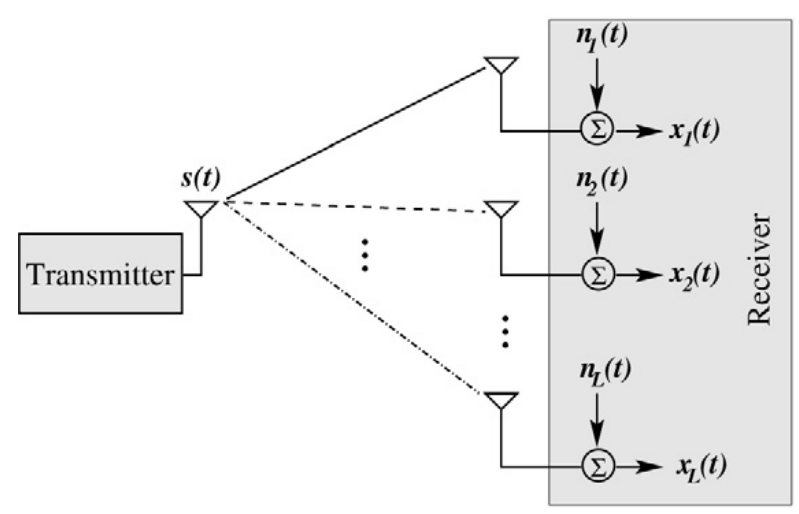

Fig. 1. Single-input multiple-output system employing multiple receive antennas. 


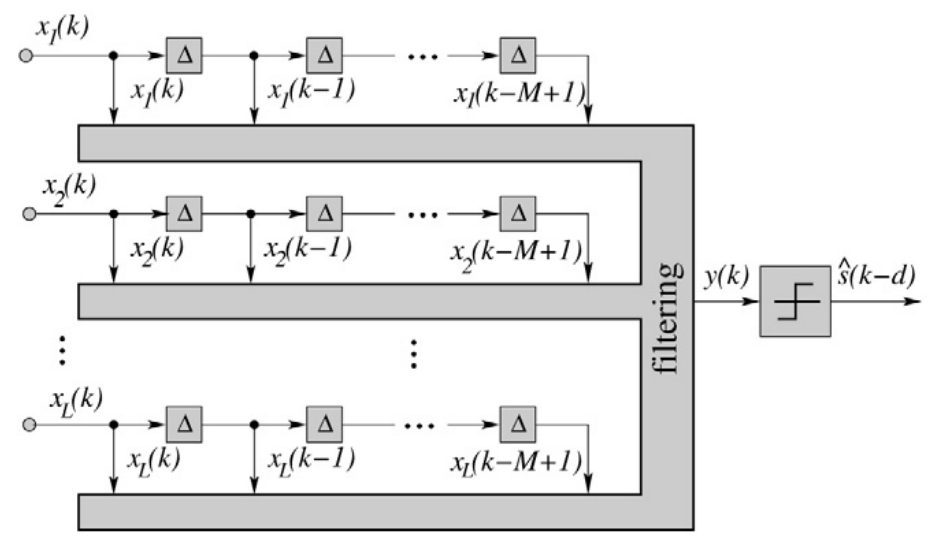

Fig. 2. Space-time equalisation structure using $\Delta$-spaced temporal filters, where $\Delta=T_{S}$ and $T_{S}$ denotes the symbol period, $M$ is the feedforward temporal filter order, and $d$ the decision delay.

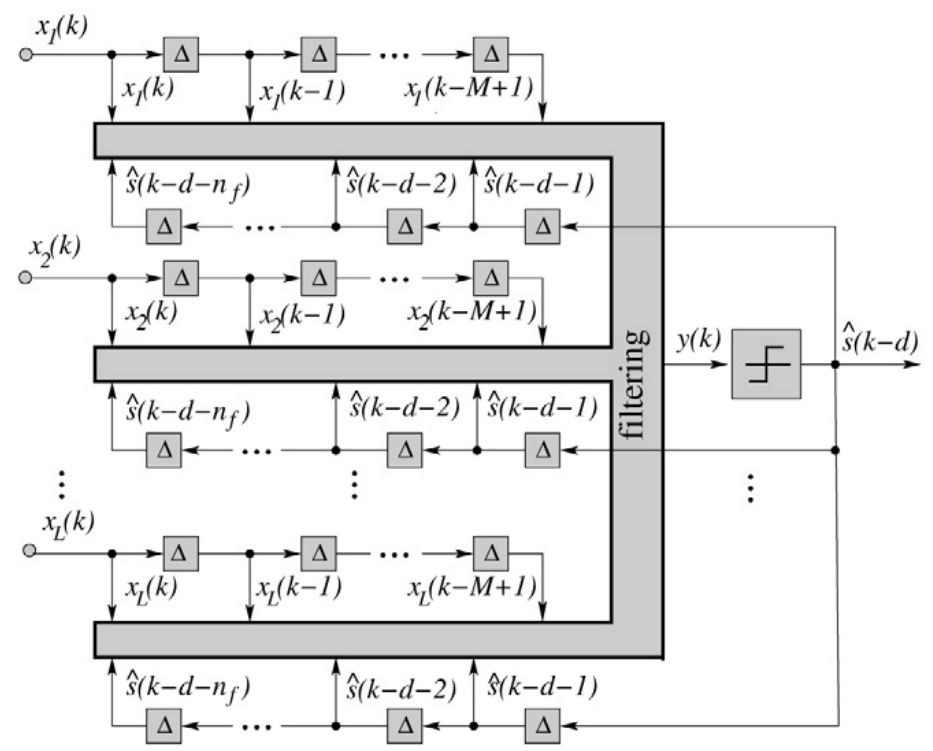

Fig. 3. Space-time decision feedback equalisation structure using $\Delta$-spaced temporal filters, where $\Delta=T_{S}$ and $T_{S}$ denotes the symbol period, $M$ is the feedforward temporal filter order, $n_{f}$ the feedback temporal filter order, and $d$ the decision delay.

generic ST-DFE structure, additionally, the feedback temporal filter order $n_{f}$ must be specified. We will define the overall signal-to-noise ratio (SNR) of the SIMO system under consideration as

$$
\mathrm{SNR}=\frac{\sigma_{s}^{2}}{L 2 \sigma_{n}^{2}} \sum_{l=1}^{L} \sum_{i=0}^{n_{c}-1}\left|c_{i, l}\right|^{2},
$$

where $\sigma_{s}^{2}=1$ is the BPSK symbol energy.

The task of the STE is to use the multi-channel output vector

$$
\mathbf{x}(k)=\left[\mathbf{x}_{1}^{T}(k) \mathbf{x}_{2}^{T}(k) \ldots \mathbf{x}_{L}^{T}(k)\right]^{T}
$$

to produce an estimate $\hat{s}(k-d)$ for $s(k-d)$, where

$$
\mathbf{x}_{l}(k)=\left[x_{l}(k) x_{l}(k-1) \ldots x_{l}(k-M-1)\right]^{T}
$$

for $1 \leqslant l \leqslant L$. Note that the equaliser's input vector $\mathbf{x}(k)$ can be expressed as

$$
\mathbf{x}(k)=\mathbf{C s}(k)+\mathbf{n}(k)=\overline{\mathbf{x}}(k)+\mathbf{n}(k),
$$


where $\mathbf{n}(k)=\left[\mathbf{n}_{1}^{T}(k) \mathbf{n}_{2}^{T}(k) \ldots \mathbf{n}_{L}^{T}(k)\right]^{T}$ with $\mathbf{n}_{l}(k)=\left[n_{l}(k) n_{l}(k-1) \ldots n_{l}(k-M+1)\right]^{T}$, the symbol vector

$$
\mathbf{s}(k)=\left[s(k) s(k-1) \ldots s\left(k-M-n_{c}+2\right)\right]^{T},
$$

and the overall CIR matrix $\mathbf{C}$ is defined as

$$
\mathbf{C}=\left[\begin{array}{c}
\mathbf{C}_{1} \\
\mathbf{C}_{2} \\
\vdots \\
\mathbf{C}_{L}
\end{array}\right]
$$

with the $M \times\left(M+n_{c}-1\right)$ CIR matrix $\mathbf{C}_{l}$ given by

$$
\mathbf{C}_{l}=\left[\begin{array}{ccccccc}
c_{0, l} & c_{1, l} & \ldots & c_{n_{c}-1, l} & 0 & \ldots & 0 \\
0 & c_{0,1} & c_{1, l} & \ldots & c_{c_{n}-1, l} & \ddots & \vdots \\
\vdots & \ddots & \ddots & \ddots & \ldots & \ddots & 0 \\
0 & \ldots & 0 & c_{0,1} & c_{1, l} & \ldots & c_{n_{c}-1, l}
\end{array}\right] .
$$

The task of the ST-DFE is to use the equaliser's input vector $\mathbf{x}(k)$ of (3) together with the equaliser's feedback vector, defined by

$$
\hat{\mathbf{s}}_{b}(k)=\left[\hat{s}(k-d-1) \hat{s}(k-d-2) \ldots \hat{s}\left(k-d-n_{f}\right)\right]^{T},
$$

to produce an estimate $\hat{s}(k-d)$ for $s(k-d)$.

\section{Adaptive Bayesian space-time equaliser}

Classical STE structure for the SIMO system is linear, and the optimal design for this linear STE is known to be the linear MBER solution [14]. However, just as in the case of the SISO channel equalisation [15-20], the optimal STE solution based on the information provided in $\mathbf{x}(k)$ is nonlinear, which will be derived in the following.

\subsection{Bayesian space-time equalisation solution}

Denote the $N_{s}=2^{M+n_{c}-1}$ legitimate combinations of $\mathbf{s}(k)$ as $\mathbf{s}_{q}, 1 \leqslant q \leqslant N_{s}$. Denote furthermore the $(d+1)$ th element of $\mathbf{s}_{q}$, corresponding to the desired symbol $s(k-d)$ as $s_{q, d}$. The noiseless SIMO channel output $\overline{\mathbf{x}}(k)$ only takes values from the finite signal state set

$$
\overline{\mathbf{x}}(k) \in \mathcal{X} \triangleq\left\{\overline{\mathbf{x}}_{q}=\mathbf{C s}_{q}, 1 \leqslant q \leqslant N_{s}\right\},
$$

which can be divided into two subsets conditioned on the value of $s(k-d)$ as

$$
\mathcal{X}^{( \pm)} \triangleq\left\{\overline{\mathbf{x}}_{q} \in \mathcal{X}, 1 \leqslant i \leqslant N_{s b}: s(k-d)= \pm 1\right\},
$$

where the size of $\mathcal{X}^{(+)}$and $\mathcal{X}^{(-)}$is $N_{s b}=N_{s} / 2$. Let the conditional probabilities of receiving $\mathbf{x}(k)$ given $s(k-d)=$ \pm 1 be $p_{ \pm}(\mathbf{x}(k))=p(\mathbf{x}(k) \mid s(k-d)= \pm 1)$. According to Bayes' decision theory [24], the optimal detection strategy is

$$
\hat{s}(k-d)= \begin{cases}1, & \text { if } p_{+}(\mathbf{x}(k)) \geqslant p_{-}(\mathbf{x}(k)), \\ -1, & \text { if } p_{+}(\mathbf{x}(k))<p_{-}(\mathbf{x}(k)) .\end{cases}
$$

Let us introduce the following real-valued Bayesian decision variable

$$
y_{\text {Bay }}(k)=f_{\text {Bay }}(\mathbf{x}(k)) \triangleq \frac{1}{2} p_{+}(\mathbf{x}(k))-\frac{1}{2} p_{-}(\mathbf{x}(k)) .
$$

The optimal detection rule (12) is equivalent to

$$
\hat{s}(k-d)=\operatorname{sgn}\left(y_{\text {Bay }}(k)\right)= \begin{cases}1, & y_{\text {Bay }}(k) \geqslant 0, \\ -1, & y_{\text {Bay }}(k)<0 .\end{cases}
$$


From the signal model (5), the decision variable (13) can be expressed as

$$
y_{\text {Bay }}(k)=\sum_{q=1}^{N_{s}} \operatorname{sgn}\left(s_{q, d}\right) \beta_{q} e^{-\frac{\left\|\mathbf{x}(k)-\overline{\mathbf{q}}_{1}\right\|^{2}}{2 \sigma_{n}^{2}}},
$$

where $\beta_{q}$ denotes the a priori probability of $\overline{\mathbf{x}}_{q}$. Since all the $\overline{\mathbf{x}}_{q}$ are equiprobable, all the $\beta_{q}$ are equal.

We now highlight a symmetric property of the Bayesian solution.

Lemma 1. The two subsets $\mathcal{X}^{(+)}$and $\mathcal{X}^{(-)}$are distributed symmetrically, that is, for any signal state $\overline{\mathbf{x}}_{i}^{(+)} \in \mathcal{X}^{(+)}$ there exists a signal state $\overline{\mathbf{x}}_{q}^{(-)} \in \mathcal{X}^{(-)}$satisfying $\overline{\mathbf{x}}_{q}^{(-)}=-\overline{\mathbf{x}}_{i}^{(+)}$.

Proof. Let $\overline{\mathbf{x}}_{i}^{(+)}=\mathbf{C} \mathbf{s}_{i}^{(+)} \in \mathcal{X}^{(+)}$. Then $-\overline{\mathbf{x}}_{i}^{(+)}=\mathbf{C}\left(-\mathbf{s}_{i}^{(+)}\right) \in \mathcal{X}^{(-)}$.

Given this symmetry property, the optimal Bayesian equalisation solution (15) can be rewritten as

$$
y_{\text {Bay }}(k)=\sum_{q=1}^{N_{s b}} \beta_{q}\left(e^{-\frac{\left\|\mathbf{x}(k)-\overline{\mathbf{x}}_{q}^{(+)}\right\|^{2}}{2 \sigma_{n}^{2}}}-e^{-\frac{\left\|\mathbf{x}(k)+\overline{\mathbf{x}}_{q}^{(+)}\right\|^{2}}{2 \sigma_{n}^{2}}}\right) \text {, }
$$

where $\overline{\mathbf{x}}_{q}^{(+)} \in \mathcal{X}^{(+)}$. The Bayesian STE solution has odd symmetry, as $f_{\text {Bay }}(-\mathbf{x}(k))=-f_{\text {Bay }}(\mathbf{x}(k))$.

\subsection{Symmetric RBF space-time equaliser design}

In the SISO equalisation case, the RBF network has been proposed to realise the Bayesian equalisation solution [16-20]. The standard RBF network however does not guarantee to posses the odd symmetry. We propose a SRBF network which has the same odd symmetry property as the Bayesian solution. The proposed SRBF network takes the following form

$$
y_{\mathrm{SRBF}}(k)=f_{\mathrm{SRBF}}(\mathbf{x}(k))=\sum_{i=1}^{N_{s b}} \theta_{i} \phi_{i}(\mathbf{x}(k)),
$$

where $f_{\mathrm{SRBF}}(\bullet)$ is a real-valued nonlinear mapping realised by the SRBF network, $\theta_{i}$ is the $i$ th real-valued RBF weight, $\phi_{i}(\bullet)$ denotes the $i$ th RBF unit, and $N_{s b}$ is the number of RBF units used. The SRBF STE makes the decision according to $\hat{s}(k-d)=\operatorname{sgn}\left(y_{\mathrm{SRBF}}(k)\right)$. In contrast to the standard RBF model, we adopt the following symmetric RBF unit

$$
\phi_{i}(\mathbf{x}) \triangleq \varphi\left(\mathbf{x} ; \mathbf{c}_{i}, \sigma_{i}^{2}\right)-\varphi\left(\mathbf{x} ;-\mathbf{c}_{i}, \sigma_{i}^{2}\right),
$$

where $\mathbf{c}_{i} \in \mathcal{C}^{L M}$ is the $i$ th complex-valued RBF centre, $\sigma_{i}^{2}$ the $i$ th real-valued RBF variance, and $\varphi(\bullet)$ the classic RBF function. In this study we adopt the Gaussian RBF function of the form

$$
\varphi\left(\mathbf{x} ; \mathbf{c}_{i}, \sigma^{2}\right)=e^{-\frac{\left\|\mathbf{x}-\mathbf{c}_{i}\right\|^{2}}{2 \sigma^{2}}} .
$$

The SRBF network (17) with the node structure (18) has an inherently odd symmetry, just as the Bayesian STE solution. A standard RBF model with the RBF node defined by $\phi_{i}(\mathbf{x}) \triangleq \varphi\left(\mathbf{x} ; \mathbf{c}_{i}, \sigma_{i}^{2}\right)$, by contrast, cannot guarantee this odd symmetry.

It is obvious that we can set all the RBF weights $\theta_{i}=\theta>0$ and all the RBF variances $\sigma_{i}^{2}=\hat{\sigma}_{n}^{2}$, where $\hat{\sigma}_{n}^{2}$ is an estimated $\sigma_{n}^{2}$. Furthermore, if we use the set of signal states $\mathcal{X}^{(+)}$as the RBF centres of this SRBF STE, then it realises exactly the optimal Bayesian performance. It can be seen that the key to realise the optimal Bayesian STE solution is to realise the optimal RBF centre set, i.e., $\mathcal{X}^{(+)}$. A simple and effective means of realising this optimal RBF centre set is to identify the SIMO CIRs using the LMS algorithm, showing as follows:

$$
\hat{c}_{i, l}(k)=\hat{c}_{i, l}(k-1)+\mu_{c} \epsilon_{l}(k) s(k-i), \quad 0 \leqslant i \leqslant n_{c}-1,
$$




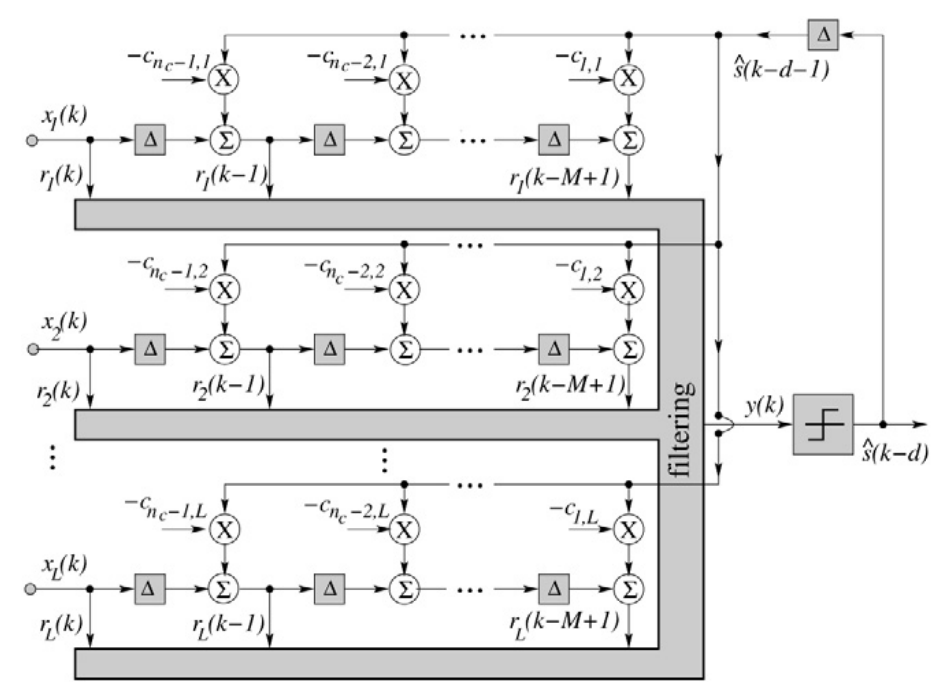

Fig. 4. Equivalent translated space-time decision feedback equalisation structure using $\Delta$-spaced temporal filters, where $\Delta=T_{S}$ and $T_{S}$ denotes the symbol period. The ST-DFE structure parameters are chosen as decision delay $d=n_{c}-1$, feedforward temporal filter order $M=n_{c}$ and feedback temporal filter order $n_{f}=n_{c}-1$, where $n_{c}$ is the SIMO CIR length.

for $1 \leqslant l \leqslant L$, where $\mu_{c}$ is the step size, and

$$
\epsilon_{l}(k)=x_{l}(k)-\sum_{i=0}^{n_{c}-1} \hat{c}_{i, l}(k-1) s(k-i) .
$$

From the estimated SIMO channel matrix $\hat{\mathbf{C}}$, it is straightforward to calculate the optimal RBF centre set, namely, $\mathcal{X}^{(+)}$. Alternatively, the clustering methods can be used to directly identify the optimal RBF centre set $[17,25,26]$. However, the method of identifying the SIMO CIRs generally requires a much shorter training sequence than the clustering approach.

The estimated noise variance $\hat{\sigma}_{n}^{2}$ is used as the RBF variance. We point out that the performance of the SRBF STE is not sensitive to the value of the RBF variance used, and there exists a large range of the RBF variance values which enable the SRBF STE to achieve the optimal Bayesian performance. This will be demonstrated in the simulation study. This robustness to the value of the RBF variance is a consequence of the Bayesian equalisation solution's robustness to the noise variance $\sigma_{n}^{2}$ used [20].

\section{Adaptive Bayesian space-time decision feedback equaliser}

As mentioned previously in Section 2, the ST-DFE uses the equaliser's input vector $\mathbf{x}(k)$ of (3) and the equaliser's feedback vector $\hat{\mathbf{s}}_{b}(k)$ of $(9)$ to detect the transmitted symbol $s(k-d)$. We will choose the ST-DFE structure's parameters as follows: $d=n_{c}-1, M=n_{c}$ and $n_{f}=n_{c}-1$. This choice may not always be optimal. However, for the SISO case, this particular choice of the DFE structure's parameters is sufficient for guaranteeing that the subsets of noise-free signal states are always linearly separable and therefore they guarantee an adequate performance [22]. We will prove that this linearly separable property is also valid for the ST-DFE structure (see Lemma 2).

We first show that the decision feedback can be viewed as a space translation, and in the translated observation space the ST-DFE is "reduced" to the STE. Using $M=n_{c}$ and $d=n_{f}=n_{c}-1$, the received signal vector $\mathbf{x}_{l}(k)$ of the $l$ th channel can be expressed as

$$
\mathbf{x}_{l}(k)=\mathbf{C}_{F_{l}} \mathbf{s}_{f}(k)+\mathbf{C}_{B_{l}} \mathbf{s}_{b}(k)+\mathbf{n}_{l}(k)
$$


where $\mathbf{s}_{f}(k)=[s(k) s(k-1) \ldots s(k-d)]^{T}, \mathbf{s}_{b}(k)=\left[s(k-d-1) s(k-d-2) \ldots s\left(k-d-n_{f}\right)\right]^{T}$, and the $M \times(d+1)$ - and $M \times n_{f}$-dimensional CIR matrices $\mathbf{C}_{F_{l}}$ and $\mathbf{C}_{B_{l}}$ are given by

$$
\mathbf{C}_{F_{l}}=\left[\begin{array}{cccc}
c_{0, l} & c_{1, l} & \ldots & c_{n_{c}-1, l} \\
0 & c_{0, l} & \ddots & \vdots \\
\vdots & \ddots & \ddots & c_{1, l} \\
0 & \ldots & 0 & c_{0, l}
\end{array}\right]
$$

and

$$
\mathbf{C}_{B_{l}}=\left[\begin{array}{ccc}
0 & \ldots & 0 \\
c_{n_{c}-1, l} & \ddots & \vdots \\
\vdots & \ddots & 0 \\
c_{1, l} & \ldots & c_{n_{c}-1, l}
\end{array}\right],
$$

respectively. Under the assumption that the past decisions are correct, $\hat{\mathbf{s}}_{b}(k)=\mathbf{s}_{b}(k)$ and the $l$ th received signal vector can be expressed as $\mathbf{x}_{l}(k)=\mathbf{C}_{F_{l}} \mathbf{s}_{f}(k)+\mathbf{C}_{B_{l}} \hat{\mathbf{s}}_{b}(k)+\mathbf{n}_{l}(k)$. Thus, the decision feedback can be viewed as a translation of the original observation space $\mathbf{x}_{l}(k)$ into a new space $\mathbf{r}_{l}(k)$

$$
\mathbf{r}_{l}(k) \triangleq \mathbf{x}_{l}(k)-\mathbf{C}_{B_{l}} \hat{\mathbf{s}}_{b}(k)=\mathbf{C}_{F_{l}} \mathbf{s}_{f}(k)+\mathbf{n}_{l}(k)=\overline{\mathbf{r}}_{l}(k)+\mathbf{n}_{l}(k) .
$$

Let us now define

$$
\mathbf{r}(k)=\left[\mathbf{r}_{1}^{T}(k) \mathbf{r}_{2}^{T}(k) \ldots \mathbf{r}_{L}^{T}(k)\right]^{T}
$$

and the $(L M) \times(d+1)$-dimensional overall CIR matrix

$$
\mathbf{C}_{F}=\left[\begin{array}{c}
\mathbf{C}_{F_{1}} \\
\mathbf{C}_{F_{2}} \\
\vdots \\
\mathbf{C}_{F_{L}}
\end{array}\right]
$$

Then

$$
\mathbf{r}(k)=\mathbf{C}_{F} \mathbf{s}_{f}(k)+\mathbf{n}(k)=\overline{\mathbf{r}}(k)+\mathbf{n}(k) .
$$

Note that the elements of $\mathbf{r}(k)$ can be computed recursively according to [22]

$$
\left\{\begin{array}{l}
r_{l}(k-i)=\Delta \cdot r_{l}(k-i+1)-c_{n_{c}-i, l} \hat{s}(k-d-1), \\
\quad \text { for } i=M-1, M-2, \ldots, 1, \\
r_{l}(k)=x_{l}(k),
\end{array}\right.
$$

where the unit delay operator $\Delta$ is defined as $\Delta \cdot r(k)=r(k-1)$. In the translated observation space $\mathbf{r}(k)$, the original ST-DFE of Fig. 3 is "translated" into the "STE" of Fig. 4.

There are $N_{s}=2^{n_{c}}=2^{d+1}$ legitimate combinations of $\mathbf{s}_{f}(k)$, again denoted as $\mathbf{s}_{q}, 1 \leqslant q \leqslant N_{s}$, for notational convenience. The noise-free part of $\mathbf{r}(k)$, namely $\overline{\mathbf{r}}(k)$, takes the value from the finite signal state set

$$
\overline{\mathbf{r}}(k) \in \mathcal{R} \triangleq\left\{\overline{\mathbf{r}}_{q}=\mathbf{C}_{F} \mathbf{s}_{q}, 1 \leqslant q \leqslant N_{s}\right\},
$$

which can be divided into two subsets conditioned on the value of $s(k-d)$ as

$$
\mathcal{R}^{( \pm)} \triangleq\left\{\overline{\mathbf{r}}_{q} \in \mathcal{R}, 1 \leqslant i \leqslant N_{s b}: s(k-d)= \pm 1\right\},
$$

where $N_{s b}=N_{s} / 2=2^{d}$.

Lemma 2. The two subsets $\mathcal{R}^{(-)}$and $\mathcal{R}^{(+)}$are linearly separable. 
Proof. We show that there exist a hyperplane $\mathbf{w}^{T} \mathbf{r}=0$ which separates $\mathcal{R}^{(-)}$from $\mathcal{R}^{(+)}$. In fact, we can choose $\mathbf{w}=\left[\begin{array}{llll}\mathbf{w}_{1}^{T} & \mathbf{w}_{2}^{T} & \ldots & \mathbf{w}_{L}^{T}\end{array}\right]^{T}$ with

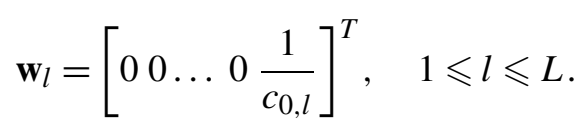

For any $\mathbf{r}_{i}^{(-)}=\mathbf{C}_{F} \mathbf{s}_{i}^{(-)} \in \mathcal{R}^{(-)}$, the last element of $\mathbf{s}_{i}^{(-)}$is -1 and

$$
\mathbf{w}^{T} \mathbf{r}_{i}^{(-)}=\sum_{l=1}^{L} \mathbf{w}_{l}^{T} \mathbf{C}_{F_{l}} \mathbf{s}_{i}^{(-)}=-L<0 .
$$

For any $\mathbf{r}_{i}^{(+)}=\mathbf{C}_{F} \mathbf{s}_{i}^{(+)} \in \mathcal{R}^{(+)}$, the last element of $\mathbf{s}_{i}^{(+)}$is +1 and

$$
\mathbf{w}^{T} \mathbf{r}_{i}^{(+)}=\sum_{l=1}^{L} \mathbf{w}_{l}^{T} \mathbf{C}_{F_{l}} \mathbf{s}_{i}^{(+)}=+L>0 .
$$

It is now apparent that the Bayesian ST-DFE solution is specified by the following "Bayesian STE" solution

$$
y_{\text {Bay }}(k)=\sum_{q=1}^{N_{s b}} \beta_{q}\left(e^{-\frac{\left\|\mathbf{r}(k)-\overline{\mathbf{r}}_{q}^{(+)}\right\|^{2}}{2 \sigma_{n}^{2}}}-e^{-\frac{\left\|\mathbf{r}(k)+\mathbf{r}_{q}^{(+)}\right\|^{2}}{2 \sigma_{n}^{2}}}\right),
$$

in the translated space $\mathbf{r}(k)$, where $\overline{\mathbf{r}}_{q}^{(+)} \in \mathcal{R}^{(+)}$and $N_{s b}=2^{d}$. An adaptive implementation of this Bayesian ST-DFE can be adopted based on the SRBF network, which involves firstly estimating the SIMO CIRs and then calculating the optimal RBF centre set using the estimated SIMO channel matrix $\hat{\mathbf{C}}_{F}$. The estimated SIMO channel taps are also used in the recursive space translation (29).

\section{Simulation study}

\subsection{STE for a fixed SIMO system}

A SIMO system with $L=4$ receive antennas was simulated, and each channel had $n_{c}=3$ taps. Table 1 lists the CIRs $\mathbf{c}_{l}^{T}=\left[\begin{array}{lll}c_{0, l} & c_{1, l} & c_{2, l}\end{array}\right], 1 \leqslant l \leqslant 4$, of this SIMO system. The actually simulated channel was normalised according to $\mathbf{c}_{l} /\left\|\mathbf{c}_{l}\right\|$ for the sake of maintaining a unity channel gain. This normalisation ensured that all the channels shared equal power but it did not change the characteristics of each channel, i.e. zero positions of the transfer function of each channel were unchanged. The STE's temporal filter order was chosen as $M=5$. For this example, the STE's decision delay $0 \leqslant d \leqslant 6$, and the optimal decision delay was found to be $d=4$. This optimal decision delay was used in the simulation. Figure 5 depicts the bit error rate (BER) of the optimal benchmark, the Bayesian STE.

The proposed SRBF based STE was next investigated. The LMS channel estimator (20) was employed to identify the $L=4$ SIMO CIRs with a step size $\mu_{c}=0.02$. The optimal RBF centre set was then computed from the estimated SIMO channel matrix. The RBF variance was set to $\sigma_{n}^{2}$. Given $\mathrm{SNR}=1 \mathrm{~dB}$, Fig. 6 shows the convergence behaviour of the LMS SIMO channel estimator averaged over 10 runs, in terms of the mean tap error

$$
\operatorname{MTE}_{l}(k)=\left\|\mathbf{c}_{l}-\hat{\mathbf{c}}_{l}(k)\right\|^{2}, \quad 1 \leqslant l \leqslant 4 .
$$

Table 1

CIRs of the simulated fixed SIMO system with $L=4$ and $n_{c}=3$. The actually simulated channel was normalised according to $\mathbf{c}_{l} /\left\|\mathbf{c}_{l}\right\|$

\begin{tabular}{lrrr}
\hline$l$ & \multicolumn{1}{l}{} & \\
\hline 1 & $-0.2+j 0.3$ & $-0.5+j 0.4$ & $0.7-j 0.6$ \\
2 & $-0.3+j 0.2$ & $0.7-j 0.1$ & $-0.5+j 0.4$ \\
3 & $-0.1-j 0.2$ & $0.6+j 0.1$ & $-0.4+j 0.3$ \\
4 & $0.1+j 0.1$ & $0.2+j 0.2$ & $-0.4-j 0.4$ \\
\hline
\end{tabular}




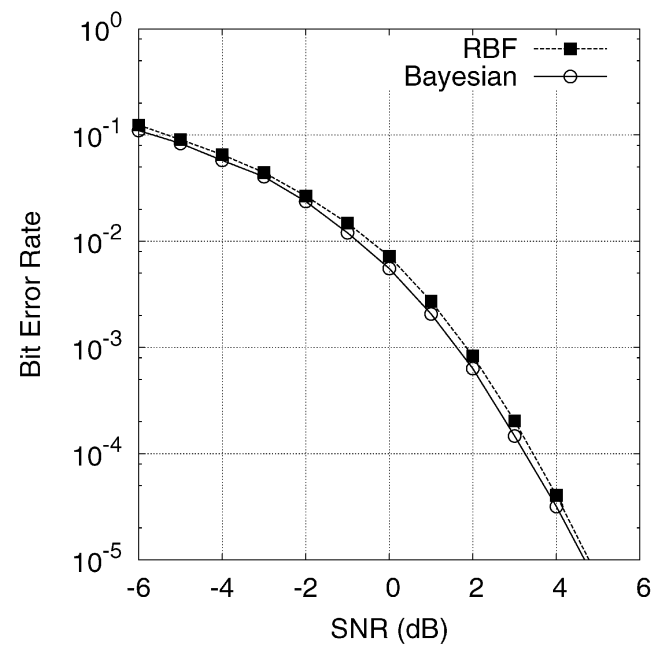

Fig. 5. Bit error rate comparison of two space-time equalisers for the fixed SIMO system listed in Table 1. Equaliser feedforward order $M=5$ and decision delay $d=4$.

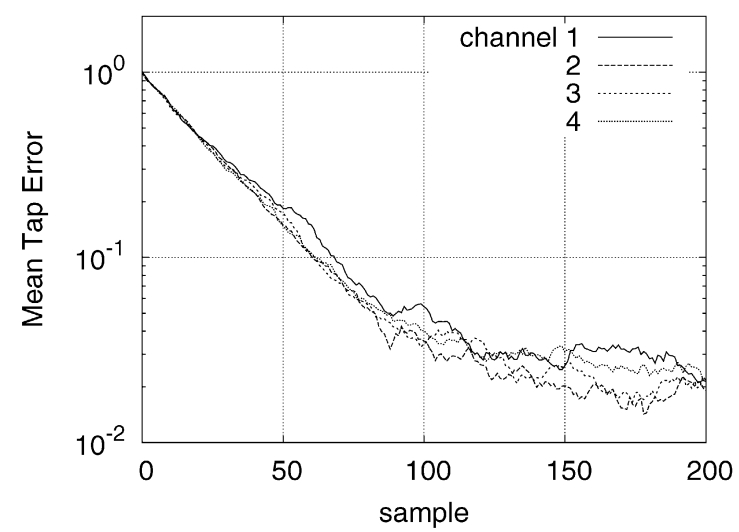

Fig. 6. Learning curve of the LMS SIMO channel estimator averaged over 10 runs for the fixed SIMO system listed in Table 1 , given SNR $=1 \mathrm{~dB}$ and the step size $\mu_{c}=0.02$.

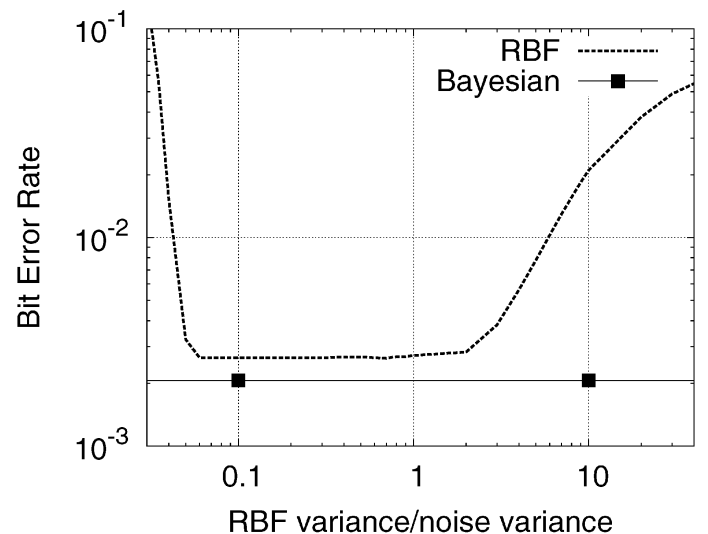

Fig. 7. Bit error rate performance of the SRBF STE as a function of the RBF variance for the fixed SIMO system listed in Table 1 , given SNR $=1 \mathrm{~dB}$ Equaliser feedforward order $M=5$ and decision delay $d=4$.

It can be seen from Fig. 6 that the LMS SIMO channel estimator had a reasonable convergence rate. The BER of the SRBF STE is plotted in Fig. 5, in comparison with the optimal Bayesian STE, where it can be seen that the SRBF STE closely approached the optimal Bayesian performance. For the same conditions in Fig. 6, Fig. 7 shows the BER 
Table 2

CIRs of the simulated fixed SIMO system with $L=4$ and $n_{c}=5$. The actually simulated channel was normalised according to $\mathbf{c}_{l} /\left\|\mathbf{c}_{l}\right\|$

\begin{tabular}{rrrrrr}
\hline$l$ & \multicolumn{1}{l}{$\mathbf{c}_{l}$} & & & \\
\hline 1 & $0.7+j 0.9$ & $-0.1+j 0.5$ & $0.6-j 0.9$ & $-0.1-j 0.4$ & $0.8+j 0.6$ \\
2 & $-0.9+j 0.2$ & $0.3-j 0.2$ & $0.5+j 0.7$ & $0.3+j 0.2$ & $-0.1+j 0.7$ \\
3 & $-0.4-j 0.4$ & $-0.1-j 0.3$ & $0.6-j 0.7$ & $-0.4-j 0.4$ & $0.5+j 0.8$ \\
4 & $0.7+j 0.3$ & $-0.8-j 0.3$ & $0.1-j 0.1$ & $0.9+j 0.3$ & $-0.1-j 0.4$ \\
\hline
\end{tabular}

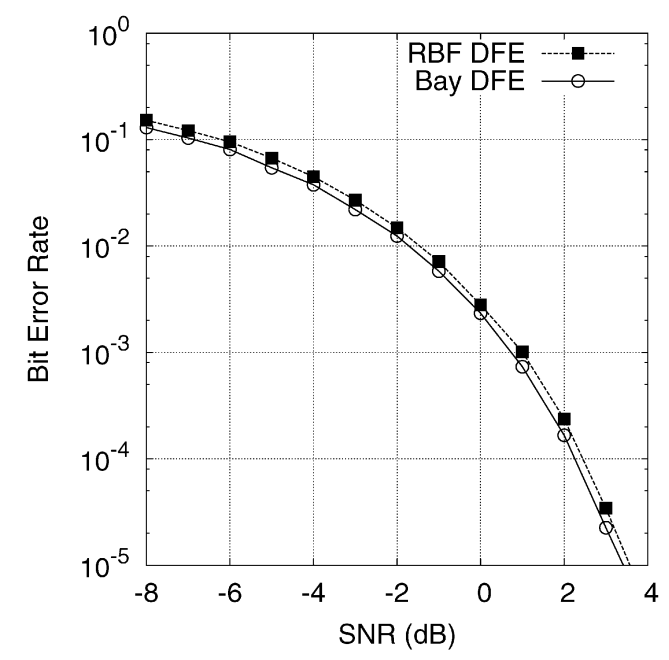

Fig. 8. Bit error rate comparison of two space-time decision feedback equalisers for the fixed SIMO system listed in Table 2. Equaliser feedforward order $M=5$, feedback order $n_{f}=4$ and decision delay $d=4$.

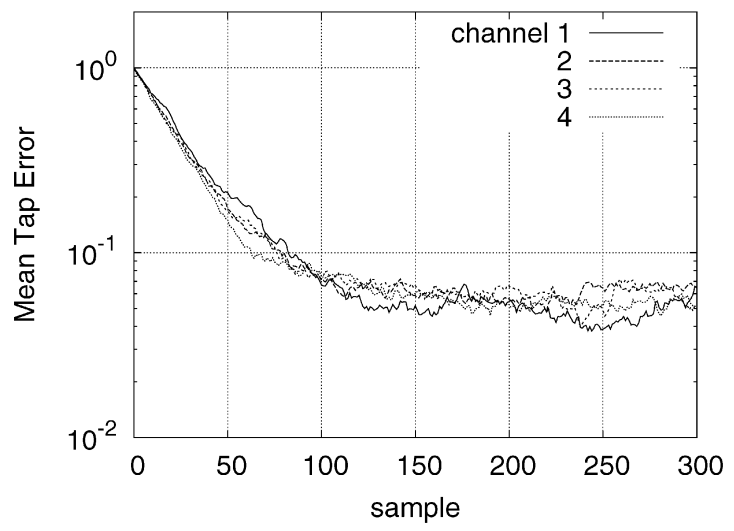

Fig. 9. Learning curve of the LMS SIMO channel estimator averaged over 10 runs for the fixed SIMO system listed in Table 2, given SNR $=0 \mathrm{~dB}$ and the step size $\mu_{c}=0.02$.

performance of the SRBF STE as a function of the RBF variance used. It can be seen from Fig. 7 that the SRBF STE approached the optimal Bayesian performance with a large range of RBF variance values.

\subsection{ST-DFE for a fixed SIMO system}

The SIMO system simulated again had $L=4$ receive antennas, but each channel had $n_{c}=5$ taps. Table 2 lists the CIRs $\mathbf{c}_{l}, 1 \leqslant l \leqslant 4$, of this SIMO system. Again the actually simulated channel was normalised according to $\mathbf{c}_{l} /\left\|\mathbf{c}_{l}\right\|$ to yielding a unity channel gain. Since the CIR length was $n_{c}=5$, the ST-DFE's structure parameters were set to the feedforward order $M=5$, feedback order $n_{f}=4$ and decision delay $d=4$. In the simulation, the detected symbols were fed back. The BER performance of the optimal Bayesian ST-DFE is depicted in Fig. 8, as the benchmark. 


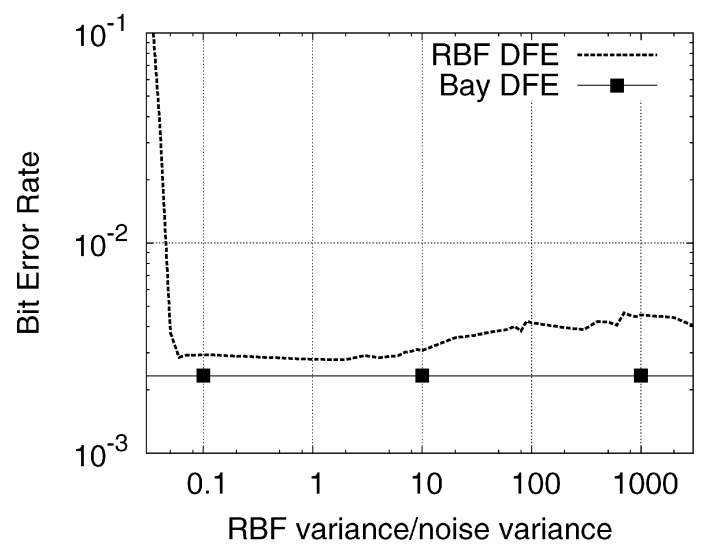

Fig. 10. Bit error rate performance of the SRBF ST-DFE as a function of the RBF variance for the fixed SIMO system listed in Table 2, given $\mathrm{SNR}=0 \mathrm{~dB}$. Equaliser feedforward order $M=5$, feedback order $n_{f}=4$ and decision delay $d=4$.

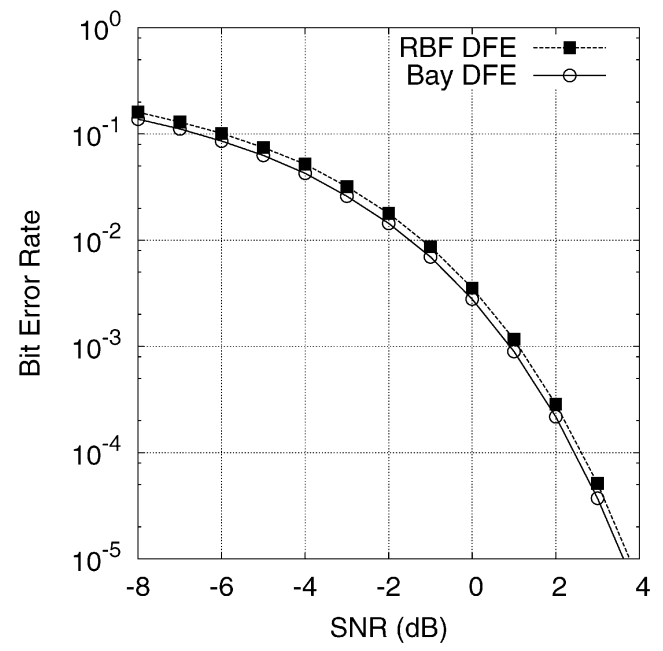

Fig. 11. Bit error rate comparison of two space-time decision feedback equalisers averaged over 50 SIMO channel realisations, where the number of receive antennas is $L=4$, each channel has a length of $n_{c}=5$, and channel taps $c_{i, l}$ are complex-valued Gaussian random processes with zero means and $E\left[\left|c_{i, l}\right|^{2}\right]=1$. Equaliser feedforward order $M=5$, feedback order $n_{f}=4$ and decision delay $d=4$.

The LMS SIMO channel estimator (20) was again employed to identify the four SIMO CIRs with a step size $\mu_{c}=0.02$. The optimal RBF centre set was then computed from the estimated SIMO channel matrix, and the estimated SIMO channel taps were used to perform the space translation (29) for the SRBF ST-DFE. The RBF variance was set to $\sigma_{n}^{2}$. With SNR $=0 \mathrm{~dB}$, Fig. 9 illustrates the learning curve of the LMS SIMO channel estimator averaged over 10 runs, in terms of the mean tap error $\operatorname{MTE}_{l}(k), 1 \leqslant l \leqslant 4$. It can be seen from Fig. 9 that the convergence rate of the LMS SIMO channel estimator was satisfactory. The BER of the SRBF ST-DFE is seen to closely approach that of the optimal Bayesian ST-DFE in Fig. 8. The robustness of the SRBF ST-DFE with respect to the value of the RBF variance used is demonstrated in Fig. 10.

\subsection{ST-DFE for a random SIMO system}

Additional simulation was performed to evaluate the proposed SRBF ST-DFE using an uncorrelated-fading SIMO system, which consisted of $L=4$ channels, each having $n_{c}=5$ taps. The channel taps $c_{i, l}, 1 \leqslant i \leqslant n_{c}$ and $1 \leqslant$ $l \leqslant L$, were complex-valued Gaussian random processes with zero means and $E\left[\left|c_{i, l}\right|^{2}\right]=1$. The ST-DFE's structure parameters were chosen to be the feedforward order $M=5$, feedback order $n_{f}=4$ and decision delay $d=4$. For each channel realisation, the LMS SIMO channel estimator (20) was used to identify the four SIMO CIRs with a step size $\mu_{c}=0.02$. The optimal RBF centre set was calculated based on the estimated SIMO channel matrix, and the estimated SIMO channel taps were used to perform the space translation (29) for the SRBF ST-DFE. The RBF 
variance was set to $\sigma_{n}^{2}$, and the detected symbols were fed back in the simulation. The performance was averaged over 50 channel realisations, and Fig. 11 compares the BERs achieved by the optimal Bayesian ST-DFE, which assumed the perfect channel state information, and the SRBF ST-DFE, which was based on the LMS SIMO channel estimates.

\section{Conclusions}

A nonlinear space-time equalisation scheme has been proposed for SIMO systems based on a novel symmetric RBF network. By exploiting the inherent symmetry of the underlying optimal Bayesian STE solution, the proposed SRBF based STE is capable of realising the optimal Bayesian equalisation performance. An adaptive implementation of this SRBF based STE has been adopted by estimating the SIMO channels using the LMS channel estimator and computing the optimal RBF centres from the resulting SIMO channel matrix estimate. Simulation results has also demonstrated that the performance of this SRBF based STE is robust with respect to the choice of the RBF variance value. The proposed adaptive nonlinear space-time equalisation scheme has then been extended to the space-time decision feedback equalisation structure. A space translation interpretation of decision feedback has been presented, and it has been shown that in the translated observation space the ST-DFE is reduced to an equivalent STE structure with a desired property that the subsets of the signal states are always linearly separable.

\section{References}

[1] G.J. Foschini, Layered space-time architecture for wireless communication in a fading environment when using multiple antennas, Bell Labs Tech. J. 1 (2) (1996) 41-59.

[2] A.J. Paulraj, C.B. Papadias, Space-time processing for wireless communications, IEEE Signal Process. Mag. 14 (6) (1997) 49-83.

[3] R. Kohno, Spatial and temporal communication theory using adaptive antenna array, IEEE Pers. Commun. 5 (1) (1998) $28-35$.

[4] J.H. Winters, Smart antennas for wireless systems, IEEE Pers. Commun. 5 (1) (1998) 23-27.

[5] A. Paulraj, R. Nabar, D. Gore, Introduction to Space-Time Wireless Communications, Cambridge Univ. Press, Cambridge, UK, 2003.

[6] A.J. Paulraj, D.A. Gore, R.U. Nabar, H. Bolcskei, An overview of MIMO communications-A key to gigabit wireless, Proc. IEEE 92 (2) (2004) 198-218.

[7] D. Tse, P. Viswanath, Fundamentals of Wireless Communication, Cambridge Univ. Press, Cambridge, UK, 2005.

[8] U. Trautwein, G. Sommerkorn, R.S. Thomä, A simulation study on space-time equalization for mobile broadband communication in an industrial indoor environment, in: Proc. VTC 1999-Spring, vol. 1, Houston, May 16-20, 1999, pp. 511-515.

[9] U. Trautwein, D. Hampicke, G. Sommerkorn, R.S. Thomä, Performance of space-time processing for ISI- and CCI-suppression in industrial scenarios, in: Proc. VTC 2000-Spring, vol. 3, Tokyo, May 15-18, 2000, pp. 1894-1898.

[10] P. Stoica, H. Vikalo, B. Hassibi, Joint maximum-likelihood channel estimation and signal detection for SIMO channels, in: ICASSP'03, vol. IV, Hong Kong, April 6-10, 2003, pp. 13-16.

[11] X. Zhu, R.D. Murch, Layered space-time equalization for wireless MIMO systems, IEEE Trans. Wireless Commun. 2 (6) (2003) $1189-1203$.

[12] S. Chen, A. Livingstone, L. Hanzo, Minimum bite-error rate design for space-time equalization-based multiuser detection, IEEE Trans. Commun. 54 (5) (2006) 824-832.

[13] S. Chen, A. Livingstone, L. Hanzo, Adaptive MBER space-time DFE assisted multiuser detection for SDMA systems, in: Proc. VTC2006Spring, vol. 1, Melbourne, May 7-10, 2006, pp. 12-16.

[14] S. Chen, A. Wolfgang, Y. Shi, L. Hanzo, Space-time decision feedback equalisation using a minimum bit error rate design for single-input multiple-output channels, IET Commun. 1 (4) (2007) 671-678.

[15] K. Abend, B.D. Fritchman, Statistical detection for communication channels with intersymbol interference, Proc. IEEE 58 (5) (1970) $779-785$.

[16] S. Chen, B. Mulgrew, Overcoming co-channel interference using an adaptive radial basis function equaliser, Signal Process. 28 (1) (1992) 91-107.

[17] S. Chen, B. Mulgrew, P.M. Grant, A clustering technique for digital communications channel equalisation using radial basis function networks, IEEE Trans. Neural Networks 4 (4) (1993) 570-579.

[18] S. Chen, B. Mulgrew, S. McLaughlin, Adaptive Bayesian equaliser with decision feedback, IEEE Trans. Signal Process. 41 (9) (1993) 29182927.

[19] S. Chen, Adaptive equalisation using neural networks, in: A.F. Murray (Ed.), Applications of Neural Networks, Kluwer, Dordrecht, 1995, pp. 241-265, chap. 10 .

[20] S. Chen, S. McLaughlin, B. Mulgrew, P.M. Grant, Adaptive Bayesian decision feedback equaliser for dispersive mobile radio channels, IEEE Trans. Commun. 43 (5) (1995) 1937-1946.

[21] S. Chen, A. Wolfgang, S. Benedetto, P. Dubamet, L. Hanzo, Symmetric radial basis function network equaliser, in: Proc. NEWCOM-ACoRN Joint Workshop, Vienna, September 20-22, 2006, CDROM 5 pages.

[22] S. Chen, B. Mulgrew, E.S. Chng, G. Gibson, Space translation properties and the minimum-BER linear-combiner DFE, IEE Proc. Commun. 145 (5) (1998) 316-322.

[23] S. Chen, S. McLaughlin, B. Mulgrew, Complex-valued radial basis function network, Part II: Application to digital communications channel equalisation, Signal Process. 36 (1994) 175-188. 
[24] R.O. Duda, P.E. Hart, Pattern Classification and Scene Analysis, Wiley, New York, 1973.

[25] C. Chinrungrueng, C.H. Séquin, Optimal adaptive $\kappa$-means algorithm with dynamic adjustment of learning rate, IEEE Trans. Neural Networks 6 (1) (1995) 1873-1896.

[26] S. Chen, Nonlinear time series modelling and prediction using Gaussian RBF networks with enhanced clustering and RLS learning, Electron. Lett. 31 (2) (1995) 117-118.

Sheng Chen received the B.Eng. degree in control engineering from China University of Petroleum, Dongying, China, in January 1982, and the Ph.D. degree in control engineering from the City University, London, UK, in September 1986. He was awarded the Doctor of Sciences (D.Sc.) degree by the University of Southampton, UK, in 2005. He joined the School of Electronics and Computer Science, University of Southampton, UK, in September 1999. He previously held research and academic appointments at the University of Sheffield, the University of Edinburgh, and the University of Portsmouth, all in UK. Professor Chen's research works include wireless communications, machine learning and neural networks, finite-precision digital controller design, and evolutionary computation methods. He has published over 300 research papers. In the database of the world's most highly cited researchers, compiled by Institute for Scientific Information (ISI) of the USA, Dr. Chen is on the list of the highly cited researchers in the engineering category.

Sheng Liu received the M.Sc. degree in wireless communications with distinction from the University of Southampton, UK, in 2006. He is currently pursuing his Ph.D. degree at the Optoelectronics Research Centre, the University of Southampton.

Lajos Hanzo received his Master degree in electronics in 1976 and his doctorate in 1983. In 2004 he was awarded the Doctor of Sciences (D.Sc.) degree by the University of Southampton, UK. During his 30-year career in telecommunications he has held various research and academic posts in Hungary, Germany, and UK. Since 1986 he has been with the School of Electronics and Computer Science, the University of Southampton, UK, where he holds the chair in telecommunications. He has co-authored 12 John Wiley/IEEE Press books totalling about 10,000 pages on mobile radio communications, published in excess of 600 research papers, organised and chaired conference sessions, presented overview lectures and has been awarded a number of distinctions. Professor Hanzo is a Fellow of the Royal Academy of Engineering (FREng), UK. He is an IEEE Distinguished Lecturer of both the Communications Society and the Vehicular Technology Society as well as a Fellow of both the IEEE and IEE. He is a nonexecutive director of the Virtual Centre of Excellence (VCE) in mobile communications, UK, and a governor of the IEEE VT society. 